\title{
Lipid-cell interactions in human monocytes investigated by doubly-resonant coherent anti-Stokes Raman scattering microscopy
}

\author{
Tyler Weeks, ${ }^{a}$ Iwan Schie, ${ }^{a}$ Laura J. den Hartigh, ${ }^{b}$ John C. Rutledge, ${ }^{\text {a,b }}$ and Thomas Huser ${ }^{a, b}$ \\ aUniversity of California, Davis, NSF Center for Biophotonics Science and Technology, Sacramento, California 95817 \\ bUniversity of California, Davis, Department of Internal Medicine, Sacramento, California 95817
}

\begin{abstract}
We demonstrate that doubly-resonant coherent anti-Stokes Raman scattering can provide enhanced and highly specific contrast for molecules containing unique Raman-active small molecular groups. This combination provides contrast for molecules that can otherwise be difficult to discriminate by Raman spectroscopy. Here, human monocytes were incubated with either deuterated oleic acid or 17-octadecynoic acid (a fatty acid with an end terminal acetylene group). The carbon-deuterium stretching vibration of the deuterated fatty acid, as well as the unique alkyne stretching vibration of the alkyne-containing fatty acid, were used to provide contrast for these exogenous free fatty acids. The combination of these unique modes with the common aliphatic carbon-hydrogen stretching vibration inherent to all fatty acid allowed for doubly-resonant detection of these unique molecules and enabled us to detect the presence of these lipids in areas within a cell where each molecular resonance by itself did not generate sufficient signal. @ 2011 Society of Photo-Optical Instrumentation Engineers (SPIE). [DOI: 10.1117/1.3544585]
\end{abstract}

Keywords: Raman scattering; coherent Raman scattering; nonlinear microscopy; lipotoxicity.

Paper 10314RR received Jun. 9, 2010; revised manuscript received Dec. 31, 2010; accepted for publication Jan. 3, 2011; published online Feb. 14, 2011.

\section{Introduction}

It is well known that the interaction between cells and dietary and blood lipids plays a key role in the pathogenesis of many diseases. In fact, it has been shown that a number of eukaryotic cells, ${ }^{1}$ such as adipocytes, ${ }^{2}$ hepatocytes,${ }^{3}$ and pancreatic $\beta$ cells, ${ }^{4}$ develop lipid accumulations in the form of lipid droplets when incubated with fatty acids. Such fundamental lipid-cell interactions have been identified as the main cause of metabolic diseases, such as nonalcoholic fatty liver disease. ${ }^{5-7}$ More recently, monocytes have also been observed to develop lipid droplets in response to direct exposure to a combination of triglyceride-rich lipoprotein (TGRL) lipolysis products consisting of lipoprotein remnant particles, diglycerides, monoglycerides, phospholipids, and free fatty acids. ${ }^{8-11}$ Production of TGRL lipolysis products in high physiological to pathophysiological concentrations of TGRL lipolysis products has been linked to vascular inflammation and atherosclerosis. ${ }^{6,8,10,11} \mathrm{Ob}$ taining a better understanding of the biogenesis of lipid droplets and the steps leading to related cellular lipid accumulations in vitro and in vivo will ultimately allow us to devise therapies for the avoidance and/or treatment of many lipid-related disorders and diseases.

Traditionally, hematoxylin and eosin (H\&E) staining has been used to identify lipid inclusions by histopathology. Here, lipid droplets simply appear as circular cavities in the stained tissue section. Alternatively, lipid droplets can be better visualized by incubating samples with hydrophobic fluorescent dyes, such as Oil-Red $\mathrm{O}$, because these dyes preferentially integrate into hydrophobic regions such as lipid droplets. While these are

Address all correspondence to: Thomas Huser, University of California, Davis, NSF Center for Biophotonics Science and Technology, Sacramento, California 95817.E-mail: trhuser@ucdavis.edu. very useful and widely utilized techniques they lack the ability to determine the chemical composition of lipid droplets and therefore may not provide sufficient information for studying lipid-cell interactions. Label-free microscopies based on vibrational molecular spectroscopy, such as Raman scattering, on the other hand, can provide very high spatial resolution and high spectral resolution which enables the chemically specific imaging and discrimination of different classes of lipids, e.g., saturated versus unsaturated fatty acids, ${ }^{12-15}$ in vitro and in vivo. Coherent anti-Stokes Raman scattering (CARS) microscopy has been shown to be particularly useful for imaging and analyzing lipid droplets in cells, especially when coupled with traditional Raman spectroscopy. ${ }^{3,15}$ Unfortunately, despite the high spectral resolution offered by Raman spectroscopy, its ability to distinguish different fatty acids in vitro is limited since many lipid spectra differ only in relative peak intensities. These similarities make the analysis of complex lipid droplets composed of many different types of lipids rather difficult. This ability to discriminate lipids is all the more important in the case of intracellular lipid droplets formed after lipid-cell interactions, where proper mapping of the distribution of specific exogenous lipids and an analysis of their biochemical effects within the cell may be critical. The use of modified lipids that provide specific and unique Raman-active vibrational signatures, e.g., deuterated lipids, or alkyne-modified lipids, ${ }^{15,16}$ can provide the needed specificity. The inclusion of such unique molecular bonds is also often referred to as "Raman labeling."

Raman labeling describes minor chemical modifications that result in a biologically unique Raman signature but will otherwise not significantly change the biological or chemical

$1083-3668 / 2011 / 16(2) / 021117 / 5 / \$ 25.00$ (C) 2011 SPIE 
nature of the labeled molecule. This is particularly important for small molecules, such as fatty acids, sugars, and peptides, which, when labeled with fluorescent moieties, will be significantly altered and may no longer exhibit their particular biochemical function. Also, fluorescence is a rather short-lived process, limited by permanent photobleaching, which only allows for rather limited windows of observation of biological samples. Modifications that alter the Raman signature of a molecule can include isotope substitutions as well as bond substitutions. The results of these modifications typically present themselves as new vibrational peaks or peak shifts in the resulting Raman spectra. Ideally, such modifications will lead to new peaks in the 1800 to $2700 \mathrm{~cm}^{-1}$ region, which is a spectral region with few, if any, vibrational modes for most biomolecules, thus providing excellent specificity for the labeled molecule. Here, we demonstrate that Raman labels, detected and amplified by doubly-resonant CARS (DR-CARS), ${ }^{16,17}$ can provide specific contrast for exogenous lipids. Specifically, we demonstrate this effect on human monocytes, which, when activated by exposure to triglyceride-rich lipoprotein lipolysis products, can injure monocytes in blood and increase monocyte binding to vascular endothelial cells and platelets, two of the cardinal interactions involved in the development of atherosclerotic cardiovascular disease. ${ }^{11}$ Our demonstration suggests that the combination of DR-CARS with Raman labels may be a particularly suitable tool for studying lipid-cell interactions based on the higher sensitivity and specificity afforded by this technique.

\section{Materials and Methods}

\subsection{Preparation of Human Monocytes}

THP-1 human monocytes were purchased from American Type Culture Collection and maintained in suspension between $5 \times 10^{4}$ and $8 \times 10^{5}$ cells $/ \mathrm{ml}$ in RPMI 1640 medium supplemented with $2 \mathrm{mM}$ L-glutamine and containing $10 \mathrm{mM}$ HEPES [4-(2hydroxyethyl)-1-piperazineethanesulfonic acid], $1 \mathrm{mM}$ sodium pyruvate, $4.5 \mathrm{~g} / \mathrm{L}$ glucose, $1.5 \mathrm{~g} / \mathrm{L}$ bicarbonate, $10 \%$ fetal bovine serum, and $0.05 \mathrm{mM} 2$-mercaptoethanol. Monocytes were incubated in $5 \% \mathrm{CO}_{2}$ and $95 \% \mathrm{O}_{2}$ at $37^{\circ} \mathrm{C}$ during growth and treatment. THP-1 monocyte treatments were conducted at a cell density of $1 \times 10^{6}$ cells $/ \mathrm{ml}$ for the times indicated. Monocyte viability was monitored after all treatments using trypan blue exclusion, and remained above $90 \%$ for all treatments. THP1 cells were treated with the synthetic fatty acids deuterated oleic acid (Cambrige Isotope Labs) or 17-octadecynoic acid (Sigma-Aldrich), at $150 \mu \mathrm{M}$ each, for $3 \mathrm{~h}$. This concentration is representative of the physiological fatty acid concentration in blood during the post-prandial phase, approximately $3 \mathrm{~h}$ after consumption of a moderately high-fat meal. These free fatty acids were first dissolved in phosphate buffered saline solution, then added to the cell's growth medium. The existence of lipid droplets was independently confirmed by differential interference contrast microscopy using a $40 \times$ objective or phase contrast microscopy using a $60 \times$ objective.

\subsection{Imaging}

Two different Raman labels were used to provide contrast for exogenous lipids. The first was isotope substitution by deuter- ation of oleic acid (dOA - Oleic Acid 9,10-D2, Cambridge Isotope), which provided a unique Raman signature at $\sim 2200$ $\mathrm{cm}^{-1}$, and the second was bond substitution by the incorporation of an acetylene group into an otherwise saturated fatty acid similar to palmitic acid (17-ODYA - 17-Octadecynoic Acid, Sigma-Aldrich), which provided a unique Raman signature at $\sim 2100 \mathrm{~cm}^{-1}$, see Fig. 1(a). It should be noted that the alkyne-modification results in a significantly more intense Raman peak even though it represents just a single bond modification, whereas two hydrogen bonds are substituted in the deuterated oleic acid. As described above, monocytes were incubated with either dOA or 17-ODYA, fixed, and then allowed to settle on a glass coverslip for high-resolution DR-CARS imaging. Images of the treated monocytes were taken with a doubly-resonant CARS microscope that utilizes a $1064 \mathrm{~nm}$ ND:Vanadate laser with 7 ps pulses (HighQ Laser) as primary laser. ${ }^{18}$ The initial beam is divided into three components, two of which synchronously pump two optical parametric oscillators (OPO's) that are tunable from 780 to $920 \mathrm{~nm}$ (Levante, APE). The three beams, $L_{1}$ from OPO $1, L_{2}$ from OPO 2 , and $L_{3}$ from the ND:Vanadate laser, are then recombined before entering the microscope, providing four detectable four-wave mixing signals, including two CARS signals, a three-color CARS signal, and a DR-CARS signal. ${ }^{17}$ In DR-CARS, three nondegenerate input photons follow two different parametric mixing pathways

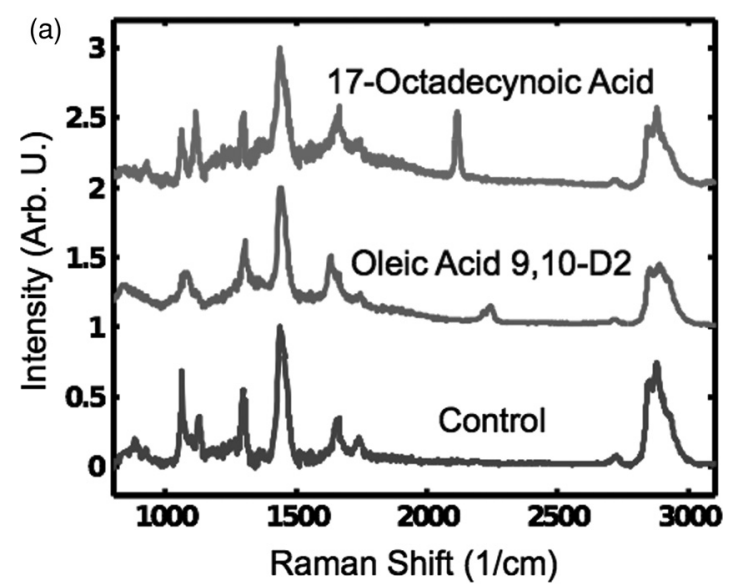

(b)

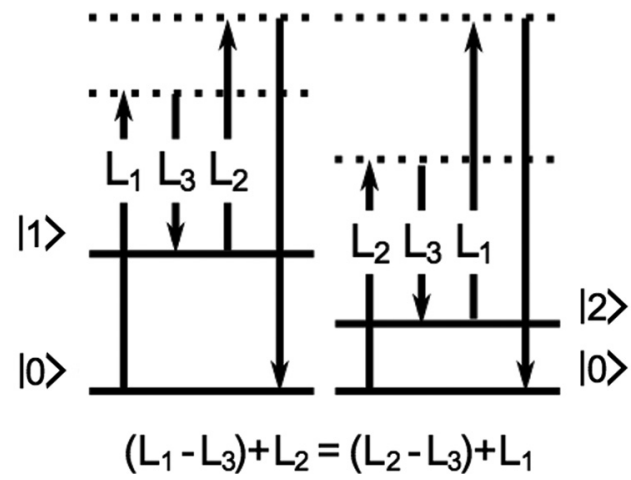

Fig. 1 (a) Raman spectra of lipid droplets within monocytes treated with 17-ODYA (top), deuterated oleic acid (middle), and naturally occurring lipid droplets (phosphate-buffered saline-no treatment, control, bottom). (b) Energy diagram of the DR-CARS process indicating that both parametric pathways result in the same signal frequency but probe different Raman resonances. 

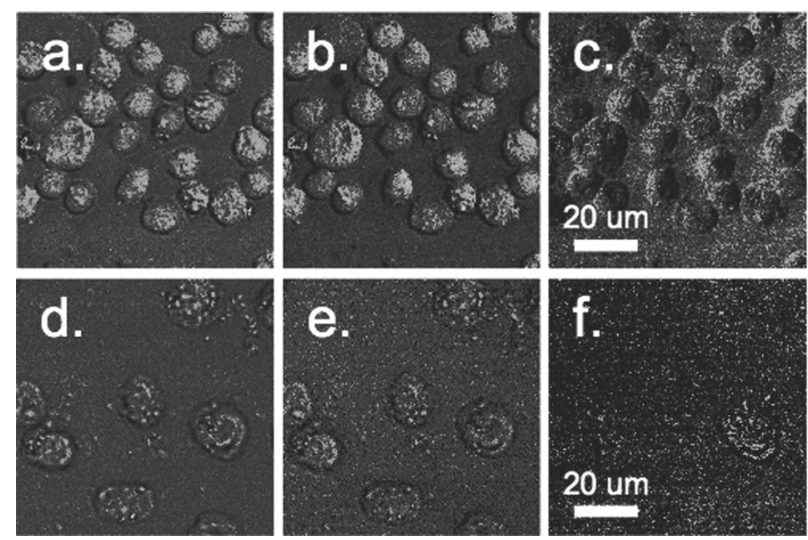

Fig. 2 (a) DR-CARS image of THP-1 monocytes incubated with deuterated oleic acid, resonant with both the $\sim 2200 \mathrm{~cm}^{-1} \mathrm{CD}$ stretch and the $2845 \mathrm{~cm}^{-1} \mathrm{CH}_{2}$ stretch. (b) CARS image of the same monocytes, resonant with just the $2845 \mathrm{~cm}^{-1} \mathrm{CH}_{2}$ stretch. (c) Difference image between (a) and (b) highlighting contrast for the $\mathrm{CD}$ stretch resonance. (d) DR-CARS image of monocytes incubated with 17-Octadecynoic acid, resonant with both the $\sim 2100 \mathrm{~cm}^{-1}$ alkyne stretch and the $2845 \mathrm{~cm}^{-1} \mathrm{CH}_{2}$ stretch. (e) CARS image of the same monocytes, resonant with just the $2845 \mathrm{~cm}^{-1} \mathrm{CH}_{2}$ stretch. (f) Difference between (d) and (e) highlighting the contrast for the alkyne stretch resonance. Note the intensity scales for $(c)$ and $(f)$ have been adjusted to highlight contrast for the lipids.

that probe distinct Raman resonances while resulting in the same signal photon, see Fig. 1(b). The OPO's were tuned such that the combination of $L_{1}$ and $L_{3}$ probed the $2845 \mathrm{~cm}^{-1} \mathrm{CH}_{2}$ stretch and the combination of $L_{2}$ and $L_{3}$ probed either the $\sim 2100 \mathrm{~cm}^{-1}$ alkyne stretch or the $\sim 2200 \mathrm{~cm}^{-1} \mathrm{CD}$ stretch. This tuning resulted in CARS signals that were resonant with each vibration individually and a DR-CARS signal that was resonant with both. For DR-CARS imaging of monocytes the laser powers are adjusted to $40 \mathrm{~mW}$ (OPO1, pump 1), $11 \mathrm{~mW}$ (OPO2, pump 2), and $12 \mathrm{~mW}$ (Nd-Vanadate laser, probe). Initially, images are scanned at an image size of $80 \mu \mathrm{m} \times 80 \mu \mathrm{m}$ at $256 \times 256$ pixels with typical pixel dwell times of $1 \mathrm{~ms}$ to identify interesting parts of a sample. The zoomed parts of the initial image are then rescanned with $256 \times 256$ pixels and image sizes according to the scale bars shown in each figure, respectively. Spontaneous Raman spectra are acquired with an integration time of $10 \mathrm{~s} / \mathrm{spectrum}$, while DR-CARS signal spectra are acqiured at $0.5 \mathrm{~s} /$ spectrum. By taking the difference between the DR-CARS (resonant with Raman label and $2845 \mathrm{~cm}^{-1} \mathrm{CH}_{2}$ stretch) and CARS (resonant with $2845 \mathrm{~cm}^{-1} \mathrm{CH}_{2}$ stretch) images, signal contributions from the Raman labels were extracted and highlighted. ${ }^{16,17}$

\section{Results and Discussion}

Images of monocytes incubated with 17-ODYA and monocytes incubated with dOA both exhibit a large number of lipid droplets that appear to reside in the cytoplasm of the cells, see Figs. 2(a), 2(b), 2(d), and 2(e). DR-CARS images [Figs. 2(a) and 2(d)] provide contrast similar to CARS images [Figs. 2(b) and 2(e)] because the labeled fatty acids provide both the Raman label and a contribution to the $2845 \mathrm{~cm}^{-1} \mathrm{CH}_{2}$ resonance. However, the difference images, obtained by subtracting a normalized $\mathrm{CH}_{2}$ CARS image from the corresponding DR-CARS image, reveal enhanced contrast for the Raman label for both groups of cells, (a)
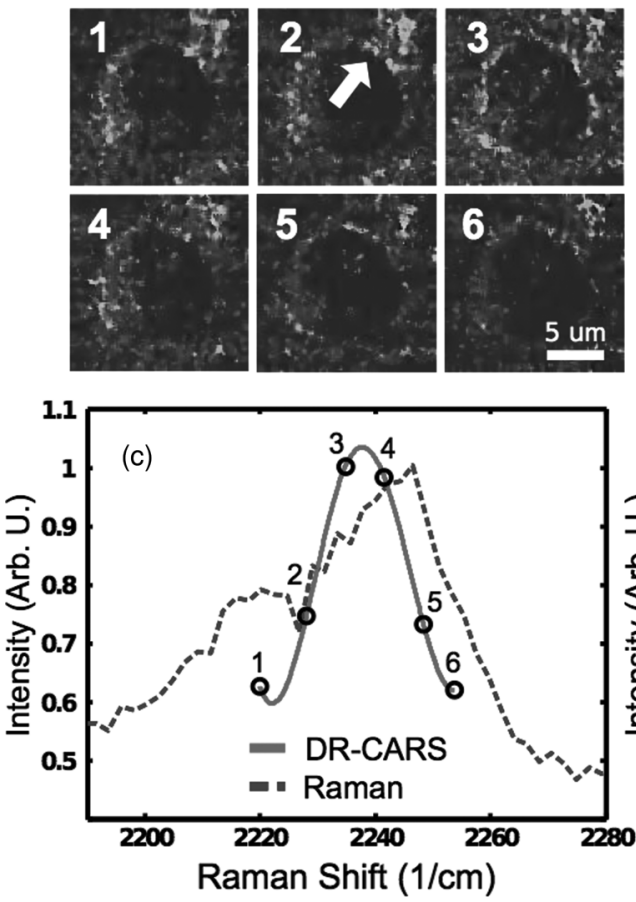

(b)
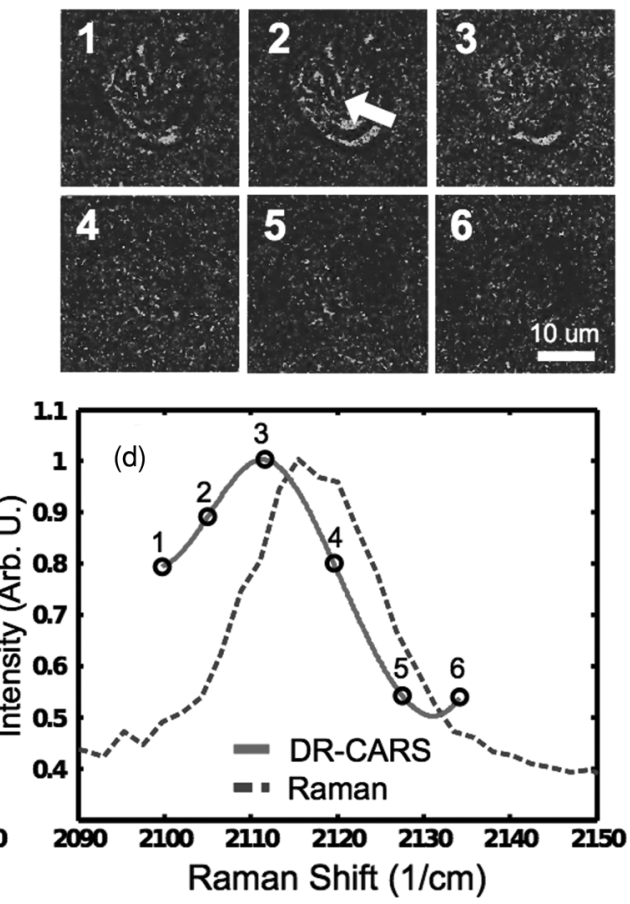

Fig. 3 (a) Difference images of monocytes incubated with deuterated oleic acid as the DR-CARS system was tuned across the CD stretch resonance. (b) Difference images of monocytes incubated with 17-ODYA as the system was tuned across the alkyne stretch resonance. (c) Comparison of DR-CARS intensities derived from the average intensity across the entire interior of the cell in each image in (a) and the spontaneous Raman peak for the CD resonance. (d) Comparison of DR-CARS intensities derived from the average intensity across the entire interior of the cell in each image in (b) and the spontaneous Raman peak for the alkyne resonance. 
see Figs. 2(c) and 2(f). Interestingly, although the deuterated oleic acid is present in the intracellular droplets, the bright rings around each cell indicate that much of this exogenous lipid may be located in the cytoplasm near the plasma membrane, see Fig. 2(c). Alternatively, while the monocytes treated with 17-ODYA also appear to develop a limited number of lipid droplets, only a small number of cells seem to incorporate the exogenous saturated free-fatty acid into the intracellular lipid droplets. Here we do not observe any notable lipid concentration near the plasma membrane, see Fig. 2(f). The "shadows" surrounding each cell are due to the interference of resonant and nonresonant signals near the edge of each cell.

To further validate that the measured difference images in Figs. 2(c) and 2(f) represent contrast for the respective Raman labels the system was tuned across each resonance, respectively, and compared with the spontaneous Raman signal of either the alkyne resonance or the CD resonance, see Figs. 3(a) and 3(b). In both cases, contrast for the Raman label was minimized as the system was tuned away from the resonance. When compared with the spontaneous Raman peaks, the peak intensity of the difference images corresponded to the expected maximum, see Figs. 3(c) and 3(d). The dependence of the contrast on the tuning of the system relative to either the alkyne or the $\mathrm{CD}$ stretch vibrational resonance suggests that it is indeed a result of probing the respective Raman labels.

Differences in the distribution of the Raman label between the two groups of cells may also serve to validate the observed contrast. Known lipid-cell interactions suggest that saturated fatty acids may be more toxic to cells than unsaturated fatty acids. $^{9,19,20}$ A natural response of monocytes exposed to exogenous molecules is cell activation. ${ }^{8-11}$ Here the result is the generation of intracellular lipid droplets, which may incorporate both endogenous and exogenous lipids. In particular, unsaturated lipids, such as oleic acid, are thought to enter cells by penetrating the plasma membrane through a flip-flop mechanism, which might explain the apparent high accumulation of deuterated oleic acid near the plasma membrane. However, exposure to more saturated fatty acids, such as 17-ODYA (except for the terminal group), leads to an overall higher lipotoxicity and apoptosis of cells, which we assume accounts for the perturbed morphology and relative low density of the cells observed after incubation with 17-ODYA, as seen in Fig. 2(d). The apparent rough shape of the cell shown in Fig. 3(b) may be due to a mechanism known as blebbing, which occurs in the late stages of apoptosis. While the monocytes treated with deuterated oleic acid appear to contain lipid droplets, they maintain their round shape and an intact plasma membrane typical of healthy human monocytes. Close inspection of Fig. 3(a) suggests that diffusion of unsaturated fatty acids across the plasma membrane leads to a locally higher oleic acid concentration near the plasma membrane, but lipid droplets are ultimately formed in the cytoplasm, likely by the endoplasmic reticulum as is the case in many other mammalian cells. The different entry pathway of unsaturated fatty acids might partially explain their overall lower lipotoxicity.

\section{Conclusions}

We have shown that Raman labels can provide a valuable contrast for small molecules such as lipids as they interact with cells. This contrast is generally difficult to achieve with traditional fluorescent labels without perturbing the interaction. Although Raman-based microscopies are much less sensitive than fluorescence-based microscopies, they do provide signals that are much more spectrally narrow which enable better chemical analysis of particular molecules and could also be exploited in terms of broad spectral multiplexing. Furthermore, the use of doubly-resonant CARS can serve to enhance sensitivity for the Raman label without the use of complicated heterodyning or lock-in schemes. Difference imaging, as demonstrated here, however, cannot completely remove background contributions (e.g., nonresonant background or laser fluctuations) from DRCARS images. We expect the combination of DR-CARS with a modulation and lock-in detection scheme to lead to completely background-free detection. Although the central motivation in the development of Raman microscopy has traditionally been its use as a label-free technique, we anticipate that Raman labels may become a powerful, if not necessary, complement to labelfree sources of contrast since they can provide true chemical specificity.

\section{Acknowledgments}

Tyler Weeks acknowledges support by the Lawrence Scholar Program from Lawrence Livermore National Laboratory. This work was supported in part by funding from the American Heart Association through a Grant-in-Aid to T.H., and by the National Science Foundation. The Center for Biophotonics, an NSF Science and Technology Center, is managed by the University of California, Davis, under Cooperative Agreement No. PHY 0120999. Support by the National Institutes of Health, National Cancer Institute under Grant No. 1U54CA136465-01 and the National Heart, Lung, and Blood Institute under Grant No. NHLBI HL055667 is acknowledged. Support is further acknowledged from the Richard A. and Nora Eccles Harrison Endowed Chair in Diabetes Research as well as from the Clinical Translational Science Center under Grant No. UL1 RR024146 from the National Center for Research Resources (NCRR), a component of the National Institutes of Health (NIH), and the NIH Roadmap for Medical Research.

\section{References}

1. S. Martin, and R. G. Parton, "Lipid droplets: a unified view of a dynamic organelle," Nat. Rev. Mol. Cell Biol. 7(5), 373-378 (2006).

2. J. P. Kampf. and A. M. Kleinfeld, "Is Membrane Transport of FFA Mediated by Lipid, Protein, or Both?," Physiology 22(1), 7-14 (2007).

3. Y. Akazawa, "Palmitoleate attenuates palmitate-induced Bim and PUMA up-regulation and hepatocyte lipoapoptosis," J. Hepatol. 42(4), 586-593 (2010)

4. H. J. Welters, M. Tadayyon, J. H. B. Scarpello, S. A. Smith, and N. G. Morgan, "Mono-unsaturated fatty acids protect against [beta]cell apoptosis induced by saturated fatty acids, serum withdrawal or cytokine exposure," FEBS Lett. 560(1-3), 103-108 (2004).

5. E. Roldan-Valadez, R. Favila, M. Martínez-López, M. Uribe, and N. Méndez-Sánchez, "Imaging techniques for assessing hepatic fat content in nonalcoholic fatty liver disease," Ann. Hepatol. 7(3), 212-20 (2008).

6. P. D. Lang and W. Insull, "Lipid Droplets in Atherosclerotic Fatty Streaks of Human Aorta," J. Clin. Invest. 49(8), 1479-1488 (1970).

7. I. R. Corbin, E. E. Furth, S. Pickup, E. S. Siegelman, and E. J. Delikatny, "In vivo assessment of hepatic triglycerides in murine 
Weeks et al.: Lipid-cell interactions in human monocytes investigated by doubly-resonant ...

non-alcoholic fatty liver disease using magnetic resonance spectroscopy," Biochim. Biophys. Acta 1791, 757-763 (2009).

8. J. L. Kelley, M. M. Rozek, C. A. Suenram, and C. J. Schwartz, "Activation of human peripheral blood monocytes by lipoproteins," Am. J. Pathol. 130, 223-231 (1988).

9. M. F. Cury-Boaventura, R. Gorjao, T. M. de Lima, P. Newsholme, and R. Curi, "Comparative toxicity of oleic and linoleic acid on human lymphocytes," Life Sci. 78(13), 1448-1456 (2006).

10. A. Alipour, A. J. van Oostrom, A. Izraeljan, C. Verseyden, J. M. Collins, K. N. Frayn, T. W. Plokker, J. W. Elte, and M. Castro Cabezas, "Leukocyte activation by triglyceride-rich lipoproteins," Arterioscler. Thromb. Vasc. Biol. 28, 792-797 (2008).

11. L. J. den Hartigh, J. E. Connolly-Rohrbach, S. Fore, T. R. Huser, and J. C. Rutledge, "Fatty acids from very low-density lipoprotein Llpolysis products induce lipid droplet accumulation in human monocytes," $J$. Immunol., 184(7), 3927-3936 (2010).

12. J. W. Chan, D. Motton, J. C. Rutledge, N. L. Keim, and T. Huser, "Raman spectroscopic analysis of biochemical changes in individual triglyceride-rich lipoproteins in the pre-and postprandial state," Anal. Chem. 77, 5870-5876 (2005).

13. J. R. Beattie, S. E. J. Bell, and B. W. Moss, "A critical evaluation of Raman spectroscopy for the analysis of lipids: Fatty acid methyl esters," Lipids 39(5), 407-419 (2004).

14. C. Krafft, L. Neudert, T. Simat, and R. Salzer, "Near infrared Raman spectra of human brain lipids," Spectrochim. Acta, Part A 61(7), 1529_ 1535 (2005)

15. M. N. Slipchenko, T. T. Le, H. T. Chen, and J. X. Cheng, "High-speed vibrational imaging and spectral analysis of lipid bodies by compound Raman microscopy," J. Phys. Chem. B 113(21) 7681-7686 (2009).

16. T. Weeks, S. Wachsmann-Hogiu, and T. Huser, "Raman microscopy based on doubly-resonant four-wave mixing (DR-FWM)," Opt. Express 17(19), 17044-17051 (2009).

17. T. Weeks, I. W. Schie, S. Wachsmann-Hogiu, and T. Huser, "Signal generation and Raman-resonant imaging by non-degenerate four-wave mixing under tight focusing conditions," J. Biophoton. 3(3), 169-175 (2010).

18. I. W. Schie, T. Weeks, G. P. McNerny, S. Fore, J. K. Sampson, S. Wachsmann-Hogiu, J. C. Rutledge, and T. Huser, "Simultaneous forward and epi-CARS microscopy with a single detector by timecorrelated single photon counting," Opt. Express 16(3), 2168-2175 (2008).

19. J. E. de Vries, M. M. Vork, T. H. Roemen, Y. F. de Jong, J. P. Cleutjens, G. J. van der Vusse, and M. van Bilsen, "Saturated but not mono-unsaturated fatty acids induce apoptotic cell death in neonatal rat ventricular myocytes," J. Lipid Res. 38(7), 1384-94 (1997).

20. G. B. Gordon, "Saturated free fatty acid toxicity. II. Lipid accumulation, ultrastructural alterations, and toxicity in mammalian cells in culture," Exp. Mol. Pathol. 27(2), 262-276 (1977). 